\title{
The agro-ecologie of the soils: Concept, wine application, example of France / L'agro-écologie des terroirs : concept, application viticole, exemple de la France
}

\author{
Joël Rochard ${ }^{1}$, Carine Herbin ${ }^{1}$, et Valérie Lempereur ${ }^{2}$ \\ ${ }^{1}$ IFV (Institut Français de la Vigne et du Vin), 17 rue Jean Chandon Moët, 51200 Epernay, France \\ ${ }^{2}$ IFV (Institut Français de la Vigne et du Vin), 210 boulevard Victor Vermorel, 69400 Villefranche-Sur-Saône, France
}

\begin{abstract}
Résumé. L'agro-écologie est une façon de pratiquer l'agriculture en utilisant au mieux le fonctionnement et les ressources de la nature tout en préservant ses capacités de renouvellement. Cette démarche vise majoritairement à concilier développement agricole, protection de l'environnement et biodiversité. En France, suite à un rapport coordonné par M. Guillou, cette approche a été lancée dès la fin 2012 par le Ministère de l'Agriculture, de l'Agroalimentaire et de la Forêt avec pour tripe objectif de concilier les performances économiques et environnementales et sociales. Pour la viticulture, les enjeux portent principalement sur la réduction de l'utilisation des produits phytosanitaires. Dans ce cadre, au-delà de l'optimisation de l'application des produits phytosanitaires, les vignobles doivent développer des structures agro-écologiques favorables à la biodiversité et à la valorisation des paysages, support de l'éco-œnotourisme. Ils sont constitués, pour l'essentiel, de haies, bosquets, arbres isolés et alignés, murets, banquettes. Ce thème a fait l'objet d'un programme européen BIODIVINE (www.biodivine.eu) associé à des opérations de démonstration dans différents vignobles en France, en Espagne et au Portugal. La communication a pour objectif de définir le concept d'agro-écologie et de formaliser, à partir de l'expérience de la France, l'application aux terroirs viticoles.
\end{abstract}

\begin{abstract}
Agro-ecology is a way of practicing agriculture by as well as possible using the operation and the resources of nature while preserving its capacitances of renewal. This approach mainly aims at reconciling agricultural development, environmental protection and biodiversity. In France, following a report coordinated by Mr. Guillou, this approach was encouraged at the end of 2012 by the Ministry for Agriculture, the Agroecology and the Forest with for objective reconciling economic and social environmental performances. For the viticulture, the challenges relate mainly to the reduction of the use of the plant health products. Within this framework, beyond the tweaking of the application of the plant health products, the vineyards must develop agro-ecological structures favorable to the biodiversity and valorization of the landscapes, support of eco-winetourism. They are made up, essentially, of the hedges, thickets, trees isolated and aligned, managed tapes buffers, low walls, benches. This topic was the object of a European program BIODIVINE (www.biodivine.eu) associated with operations of demonstration in various vineyards in France, Spain and in Portugal. The communication aims to define the concept of agro-ecology and to formalize to leave the experiment France the application to the vine terroirs.
\end{abstract}

\section{Introduction}

Les pratiques d'agriculture intensive, qui se sont répandues en Europe au XXe siècle, ont permis une importante augmentation de la productivité, notamment grâce à de nombreuses innovations scientifiques et techniques. Néanmoins le changement des pratiques a eu des conséquences, notamment sur la biodiversité, la qualité des écosystèmes et les paysages par une simplification des espaces viticoles. En complément la pression socioculturelle vis-à-vis de l'utilisation des pesticides a conduit à un renforcement de réglementation.

Ainsi, plusieurs directives européennes visant à réglementer l'utilisation des produits phytopharmaceutiques ont vu le jour, accompagnées en France d'une stratégie nationale dont les plans Ecophyto qui visent à réduire progressivement l'utilisation des produits phytosanitaires tout en maintenant une agriculture économiquement performante.

Dans la problématique du développement durable, l'agriculture occupe une place importante car elle joue un rôle notable dans la mise en valeur des territoires, elle répond à un des besoins humains les plus fondamentaux. La durabilité de l'agriculture est donc essentielle. Mais la notion de développement durable, est souvent banalisé et parfois galvaudé, ce qui a conduit à l'émergence de nouveaux concepts : écologie industrielle, économie et croissance verte, économie sociale et solidaire, écodéveloppement, et plus particulièrement pour les secteurs agricoles et agroalimentaires, l'économie circulaire ainsi que l'agro-écologie.

L'économie circulaire, contrairement à une économie linéaire, a pour objectif de produire des biens et des services tout en limitant la consommation et le 


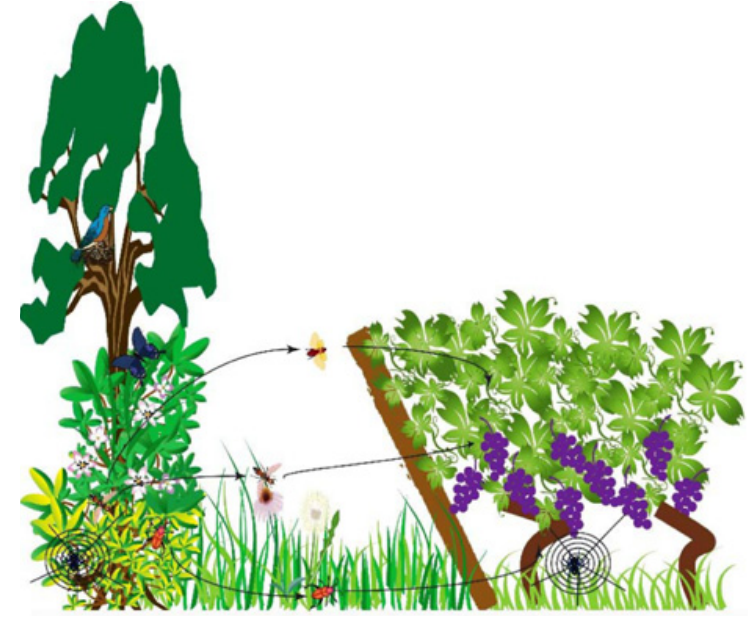

Figure 1. Les Zones Ecologiques Réservoirs sont des espaces semi-naturels implantés à proximité des vignes. Ils sont composés d'arbustes, d'arbres ou de plantes rampantes (Source : www. saumur-champigny.com).

gaspillage des matières premières, de l'eau et des sources d'énergie. Elle s'appuie notamment sur l'analyse de cycle de vie et concerne dans une certaine mesure la production agricole à l'origine de sous-produits valorisables, mais surtout les processus de transformation par une approche d'éco-conception des produits.

L'agro-écologie associe, dans une approche intégrative, l'agronomie et l'écologie pour l'ensemble de la production agricole au sein de son territoire. Cet objectif, audelà de l'optimisation de l'application des produits de traitement, impose de favoriser des mécanismes de régulation naturelle des parasites en lien avec les services écosystémiques des territoires agricoles et viticoles (Fig. 1).

En 2012, le projet agro-écologique pour la France a été présenté par le Ministère de l'Agriculture, de l'Agroalimentaire et de la Forêt dont le plan d'action très précis a été validé en 2014 et actuellement mis en œuvre. Ses objectifs principaux portent sur le développement et la diffusion de pratiques agro-écologiques afin d'assurer une agriculture performante sur le plan économique, environnemental et social, en optimisant l'utilisation des ressources et des mécanismes naturels travers plusieurs informations, la mobilisation des aides de la PAC, le développement de génétique adaptée.

« Nous devons aborder ces questions de l'écologie et de l'agriculture, non pas en les segmentant ou en les séparant, mais en les inscrivant dans des logiques de système. C'est la nouveauté de ce projet sur l'agroécologie. » Stéphane Le Foll, ministre de l'agriculture, de l'agroalimentaire et de la forêt.

Une stratégie d'agro-écologie impose de favoriser les mécanismes de régulation naturelle des parasites et de s'intéresser notamment à la biodiversité et aux composantes paysagères des terroirs. Comme le souligne J. Loyat «la biodiversité est une composante environnementale mal prise en compte, en l'absence d'une vision claire de son état et de sa dynamique. Une part importante de la biodiversité terrestre se trouve dans les espaces agricoles, et l'agriculture à un rôle déterminant dans sa gestion. L'enjeu est de concevoir et développer une « écoagriculture » qui satisfasse aux besoins croissants

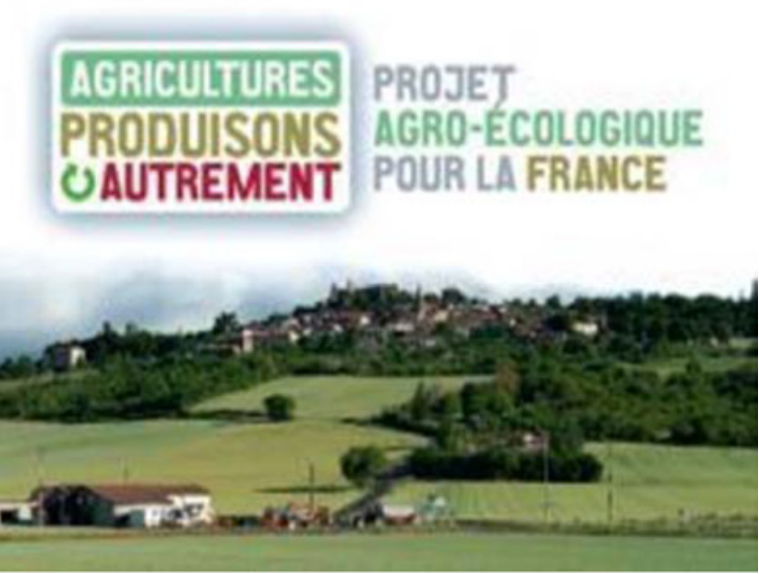

Figure 2. Plaquette 《Projet agro-écologique pour la France », Ministère de l'agriculture de l'agro-alimentaire et de la forêt, 2013.

de l'alimentation et des cultures non alimentaires, tout en préservant la diversité du vivant et en conservant la capacité d'adaptation des espèces et des écosystèmes. (... Les savoirs et savoir-faire locaux, longtemps ignorés, témoignent de la coévolution sur le très long terme des sociétés et de la biodiversité. Leur protection fait partie des stratégies pour le développement durable. Pour assurer une « bonne gouvernance de la biodiversité », il faut associer dans des actions collectives les décideurs légitimes, les scientifiques et les parties prenantes, à des échelles qui vont du niveau international jusqu'au niveau local » (Fig. 2) [5].

Un rapport piloté par Marion Guillou, publié en juin 2013, qui a associé les acteurs de la recherche et du développement ainsi que les services du Ministère de l'Agriculture, a proposé une analyse en profondeur des voies et moyens pour combiner efficacement performance économique et performance environnementale. [4]. Fondé sur l'examen d'initiatives concrètes en France et à l'étranger et sur l'expertise scientifique, ce document a constitué une étape importante car il a montré que la voie de la performance économique et environnementale était réaliste et porteuse d'avenir. En 2014, la loi d'avenir pour l'agriculture, l'alimentation et la forêt a été mise en place pour assurer un développement opérationnel de l'agro-écologie. Dans cette optique, ce texte a mis en place les groupements d'intérêt économique et environnemental (GIEE). Il permet de reconnaître des collectifs d'agriculteurs qui s'engagent dans un programme pluriannuel d'amélioration ou de consolidation de leurs pratiques, avec la triple performance. Ils peuvent ainsi bénéficier de majorations dans l'attribution des aides publiques pour accompagner leur projet agro-écologiques. Depuis mars 2015, près de 250 GIEE ont déjà été créés (Fig. 3) [6,7].

\section{Définitions et objectifs}

Le néologisme «agro-écologie » est apparu dans la littérature dans les années 30, d'abord pour désigner une discipline scientifique au carrefour de l'écologie et de l'agronomie, puis à partir des années 70 , un ensemble de pratiques et un mouvement social en réaction à l'intensification de l'agriculture [2]. 


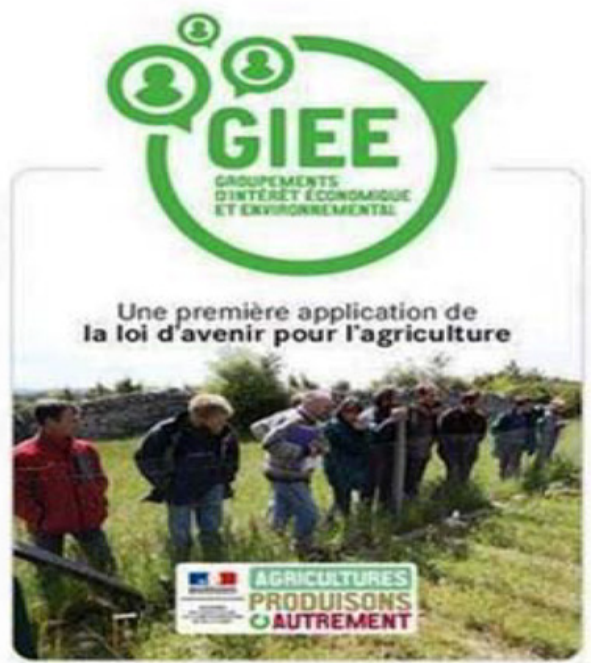

Figure 3. Plaquette « GIEE une première application de la loi d'avenir pour l'agriculture ». Ministère de l'agriculture de l'agro-alimentaire et de la forêt, 2016. http://agriculture . gouv.fr/ministere/giee-une-premiere-applicationde-la-loi-davenir-pour-lagriculture;

http://agriculture.gouv.fr/agro-ecologie-lesactions-realisees-en-2015

Ce concept peut être défini comme un ensemble disciplinaire alimenté par le croisement des sciences agronomiques (agronomie, zootechnie), de l'écologie appliquée aux agroécosystèmes et des sciences humaines et sociales [11].

D'un point de vue plus opérationnel, les systèmes de production agro-écologiques s'appuient sur les services fournis par l'écosystème et les utilisent, tout en limitant leurs impacts négatifs sur l'environnement et en cherchant à préserver les ressources naturelles de façon durable. Les pratiques sont également à adapter au cas par cas, en fonction du milieu et du contexte socio-économique. L'exploitant expérimente avec ses méthodes, et les adapte en fonction des besoins.

Au-delà des aspects liés à la production agricole sensu stricto, l'agro-écologie peut également recouvrir un sens plus large en considérant les dynamiques territoriales et les acteurs sociaux portant les fondements d'une agriculture durable, écologiquement saine, économiquement viable et socialement juste [13]. Ainsi les acceptions du terme agro-écologie sont multiples et vont bien au-delà d'un positionnement scientifique, en se référant également à des pratiques agricoles et à un mouvement social [12].

Une approche internationale commence à s'organiser autour des principes de l'agro-écologie, avec la participation d'organismes institutionnels tels que l'Organisation des Nations Unies pour l'alimentation et l'agriculture (FAO). Cette dernière a organisé en 2014 à Rome un symposium international sur l'agro-écologie pour la sécurité alimentaire et la nutrition, parrainée notamment par la France et la Suisse, prélude un programme de travail dédié sur trois ans (avec des conférences régionalisées). La FAO considère que l'agro-écologie est un moyen viable d'assurer la sécurité alimentaire mondiale, en particulier dans un contexte de changements climatiques (FAO, 2015).

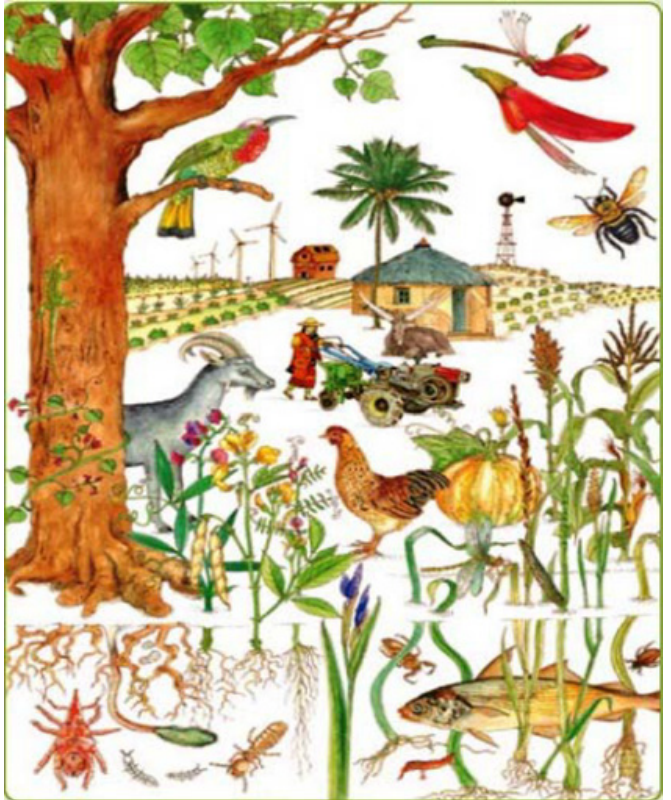

Figure 4. Symposium international sur l'agro-écologie pour la sécurité alimentaire et la nutrition, de la FAO, Rome, 2014.

«L'Agro-écologie continue de croître, à la fois dans les sciences et dans les politiques. Il s'agit d'une approche qui aidera à relever le défi de mettre un terme à la faim et à la malnutrition sous toutes leurs formes, dans le contexte de l'adaptation au changement climatique de plus en plus nécessaire », José Graziano da Silva, Directeur Général de la FAO, 2014 (Fig. 4) [3].

Un des principes de l'agro-écologie est de prendre conscience des services écosystémiques fournis par l'environnement autour et dans la parcelle agricole et de les utiliser. On cherche à optimiser ces services en limitant les apports en produits phytopharmaceutiques, en favorisant la faune du sol et en maintenant et restaurant des écosystèmes naturels diversifiés à proximité des parcelles cultivées. Parmi les services rendus par la biodiversité en milieu agricole, on retrouve la fertilisation des sols, les ressources en eau, la régulation du climat, la dégradation de molécules phytopharmaceutiques ou encore le contrôle des bio-agresseurs par la lutte biologique [10].

D'un point de vue opérationnel, les principales orientations agro-écologiques sont les suivantes :

- Travail du sol qui respecte sa structure et maintien les populations des divers micro-organismes et animaux dans les horizons du sol en veillant à sa matière organique. Un couvert végétal est recherché pour limiter l'érosion et structurer le sol.

- Accroissement de la biodiversité (intra-parcellaire, au sein de l'exploitation et du territoire) en évitant les monocultures qui ont besoin d'intrants en énergie, pesticides et engrais. L'intégration éventuelle d'animaux est également au centre des préoccupations des agriculteurs pratiquant l'agro-écologie.

- Fertilisation obtenue au moyen des engrais verts et de compost. L'objectif est le maintien d'un taux d'humus élevé assurant une fertilité durable et garantissant une alimentation hydrique plus régulière. Traitements phytosanitaires optimisés en privilégiant les produits à faible impact environnemental et éco-toxicologique. 
- Sélection des variétés les plus adaptées au contexte local.

- Optimisation de l'irrigation.

- Réduction de la consommation énergétique des itinéraires techniques.

- Aménagements parcellaires pour lutter contre l'érosion des sols et utilisation éventuelle les eaux de pluie, pour recharger les nappes phréatiques.

- Préservation, restauration, implantation de structures agro écologiques pour favoriser la biodiversité et les structures paysagères. L'agroforesterie peut également s'inscrire dans ce processus.

- Réhabilitation des savoir-faire traditionnels liés à la gestion écologique et économique des territoires.

- Pédagogie adaptée aux acteurs de terrain.

L'agro-écologie, dans son objectif de durabilité, cherche à assurer la conservation des sols et le maintien de leur productivité à long terme. Des aménagements permettent de limiter l'érosion des sols. Parmi ces structures, on peut citer les haies brise-vent, les murets de pierre ou encore le maintien d'un couvert enherbé.

Les exploitants pratiquant l'agro-écologie recherchent le maintien d'espaces naturels et d'aménagements favorisant leur conservation. Ces milieux sont des refuges pour de nombreuses espèces végétales et animales. On encourage notamment la présence de plans d'eau, de fossés, de bosquets et de talus. Ruisseaux, forêts, haies et bandes herbacées peuvent permettre de constituer des corridors écologiques favorables aux déplacements de la faune.

L'agro-écologie associe également ne lutte intégrée efficace qui passe par une bonne estimation des risques. Des réseaux de surveillance fournissent des informations sur la présence des nuisibles en fonction des cultures et permettent d'échanger avec d'autres professionnels. Les ressources techniques et économiques de l'exploitant, l'accès au soutien technique d'organismes de surveillance ou de recherche influence les mesures de protection mises en place sur l'exploitation.

http://agriculture.gouv.fr/les-fondementsde-lagro-ecologie

\section{Mise en œuvre de l'agro-écologie en viticulture}

La viticulture a subi, comme les autres secteurs agricoles, une intensification des pratiques culturales, accompagnée d'une utilisation accrue des produits de synthèse. En viticulture, les demandes du grand public renforcées par la dimension culturelle du vin et la sensibilisation des professionnels, contribuent au développement de pratiques alternatives plus respectueuses de l'environnement et de la santé $[8,10]$.

L'agro-écologie au vignoble prend notamment en compte la lutte intégrée, la mise en place d'actions et d'aménagements environnementaux, ainsi que la valorisation de la biodiversité et des milieux naturels.

La lutte intégrée fait intervenir un panel de techniques de défense alternatives, en considérant l'intervention chimique comme un dernier recours contre les maladies et les ravageurs. La prophylaxie, notamment, regroupe un ensemble de pratiques dont le but est de prévenir l'apparition, la propagation ou l'aggravation des dégâts subis par la culture. On cherche en premier lieu à diminuer la sensibilité de la vigne. Cet objectif peut être atteint en choisissant des porte-greffes et des cépages adaptés aux caractéristiques climatiques et résistants aux maladies locales, ainsi qu'en utilisant des plants garantie sains [1].

La lutte biologique réunit un ensemble de méthodes basées sur l'utilisation d'organismes vivants pour lutter contre des espèces considérées comme nuisibles. Ces auxiliaires de culture ont pour caractéristique un mode de vie entraînant la destruction des individus indésirables. On cherche ainsi à réguler les populations de ravageurs en dessous d'un seuil auquel on considère les dégâts économiques et qualitatifs comme acceptables. La mise en application de ces techniques implique d'avoir une bonne connaissance de la biologie et de l'écologie des espèces concernées, nuisibles comme auxiliaires. Il est nécessaire de prendre en compte les interactions de ces organismes entre eux, mais également avec leur environnement et les autres espèces présentes dans le milieu.

Il existe trois types de lutte biologique :

- La méthode par acclimatation consiste à apporter un auxiliaire pour lutter contre un ravageur.

- La lutte biologique par augmentation doit permettre de contrôler un ravageur indigène, soit en augmentant les effectifs de son ennemi naturel, soit en introduisant régulièrement un auxiliaire qui ne saurait se maintenir seul dans le milieu. Les organismes sont multipliés en « élevage» et sont introduits lors de lâchers « inondatifs ».

- La lutte biologique par protection a pour objectif d'attirer les auxiliaires sur les cultures en favorisant leur habitat et en adaptant les pratiques culturales. Les programmes de traitement, notamment, doivent prendre en compte les effets non intentionnels des produits phytopharmaceutiques sur la faune auxiliaire.

En complément, la lutte biotechnique correspond à des moyens de défense contre les ravageurs comprenant des stimuli physiques ou chimiques ainsi que des agents agissant sur le développement ou le comportement des organismes visés. En France, ce moyen de lutte est utilisé par confusion sexuelle pour limiter les populations d'eudémis et de cochylis. Le principe de cette méthode est de saturer artificiellement l'atmosphère du vignoble en phéromones femelles ce qui perturbe la reproduction.

La biodiversité 《fonctionnelle » mais également « naturaliste », qui favorise la protection et la valorisation des habitats naturels, se diffusent depuis quelques années auprès des viticulteurs. D'un point de vue écologique, les milieux naturels servent de refuges à une flore et une faune variée. Ils peuvent constituer des corridors écologiques pour le déplacement des espèces entre des espaces favorables plus importants. De plus, la conservation et la restauration de sites naturels sont également utiles pour mettre en avant les valeurs esthétiques et patrimoniales $\mathrm{du}$ terroir viticole en favorisant une diversification des paysages. Ces différents aspects ont été développés dans le cadre du programme européen Life BioDiVine (www.biodivine.eu). 


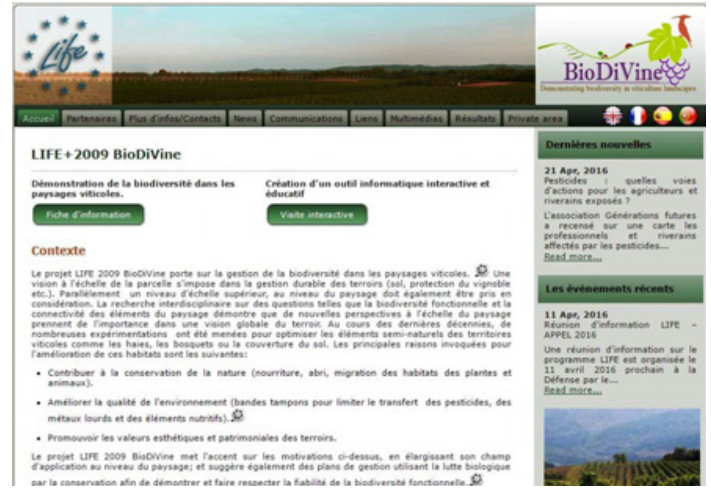

Figure 5. Site Internet du projet BioDiVine (www. biodivine.eu).

\begin{tabular}{l}
\hline Projet européen LIFE + BioDiVine : \\
Le projet BioDiVine a porté sur l'étude et la gestion \\
de la biodiversité dans les paysages viticoles. Ce projet, \\
qui a béneficié d'un financement européen LIFE + \\
$2009 / 2014$, vise à cerner l'intérêt des aménagements \\
des terroirs viticoles et l'adaptation des itinéraires \\
techniques concernant la biodiversité, le paysage et, plus \\
globalement, l'environnement. Le projet s'est concentré \\
sur la gestion de six sites expérimentaux européens : \\
Douro au Portugal, Rioja et Penedès en Espagne, un \\
site de référence, Saumur-Champigny, ainsi que les \\
appellations Saint Émilion, Limoux et Costières de \\
Nîmes et Bourgogne en France. Des actions concrètes de \\
conservation et d'aménagement ont été mises en ouvre \\
sur chaque site avec la création d'espaces semi-naturels \\
complémentaires. Parallèlement aux aménagements, une \\
protection raisonnée ou intégrée du vignoble a été \\
développée. De plus, des suivis techniques par le biais \\
de protocoles appropriés aux différentes spécialités de la \\
biodiversité et une analyse cartographique ont été réalisés \\
afin d'évaluer la portée des actions mises en œuvre \\
(www.biodivine.eu) (Figs. 5 et 6) [9].
\end{tabular}

En France, les espaces viticoles étant majoritairement composés de parcelles de dimensions réduites, les nombreux espaces interstitiels sont propices à la restauration de milieux favorables à la biodiversité, notamment des haies, des murets ou encore des bandes herbacées et fleuries. La vigne étant une culture pérenne, le vignoble constitue un paysage stable offrant des possibilités d'aménagements à long terme (Fig. 7).

Le vignoble est également favorable à la mise en place de surfaces herbacées. L'enherbement se définit comme le maintien d'un couvert végétal, naturel ou semé, permanent ou temporaire. Sa gestion doit être adaptée au contexte local (pente, types de sols, climat, etc.). Il peut être mené sur l'inter-rang, sur les chemins et les 《 tournières » à l'extrémité des parcelles, et parfois sous le rang. Il permet de limiter l'érosion de la parcelle, ainsi que l'utilisation et le ruissellement des intrants. Il favorise l'aération et la perméabilité du sol grâce aux racines de la végétation herbacée. Cette dernière réduit également le compactage provoqué par le passage des machines agricoles et constitue un apport de matière organique. Dans certaines situations, l'enherbement constitue un moyen de limiter la vigueur de la vigne. Enfin, la flore spontanée est un lieu de refuge pour la biodiversité, notamment les auxiliaires et la faune du sol. L'enherbement constitue

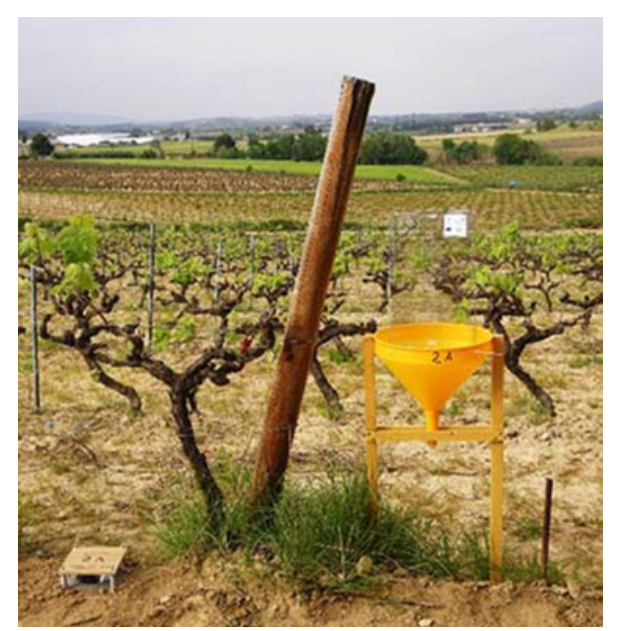

Figure 6. Evaluation de la biodiversité des arthropodes par la méthode RBA (Rapidity Biodiversity Assessment).

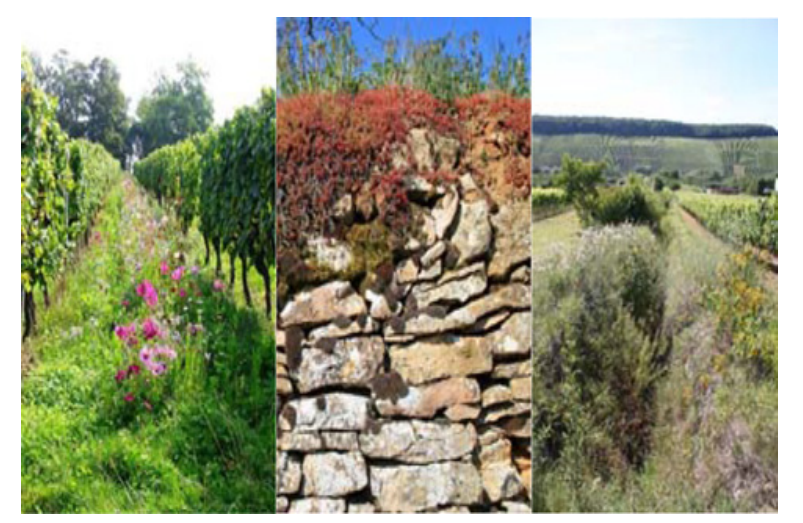

Figure 7. Quelques exemples de gestion de biodiversité dans les terroirs viticoles (jachère fleurie, muret, gestion des fossés).

alors un corridor écologique au sein même de la parcelle cultivée.

\section{Conclusion}

Au cours des précédentes décennies, par l'intensification de l'agriculture, l'agronomie s'est parfois éloignée de l'écologie. Comme le souligne J. Loyat «En une quinzaine d'années, le développement durable s'est imposé sur la scène politique et économique. Il fait désormais partie des valeurs que la communauté internationale désire promouvoir et il est devenu un élément incontournable de tout discours qui entend traiter de la société et de son avenir. Il y a là un incontestable effet de mode, mais qui plonge ses racines dans le phénomène bien réel à la fois de la finitude des ressources par rapport à des besoins qui croissent sans cesse et de l'échec de la croissance pour garantir la pérennité de nos sociétés. C'est, en dernier ressort, l'organisation de nos sociétés qui est en question, dans sa capacité à satisfaire les besoins les plus essentiels $\gg[5]$.

L'agro-écologie est une façon de concevoir des systèmes de production qui s'appuient sur les fonctionnalités offertes par les écosystèmes. Elle implique le recours à une diversité technique valorisée, par des savoir-faire locaux, en considérant l'exploitation dans son ensemble et les parcelles au sein d'un territoire. Le défi est considérable et impose une modification très significative 
des pratiques des viticulteurs. Un tel changement, même s'il est déjà amorcé, suppose un investissement lourd dans la recherche, la formation, le conseil et l'éducation.

Parallèlement aux démarches régionales et nationales, il est important que ce concept soit accompagné par les instances internationales qui peuvent fournir, dans le prolongement du développement durable, des guides de mise en œuvre de l'agro-écologie à adapter à chaque contexte local.

\section{Références}

[1] Avenard, J.-C. et coll., Manuel de production intégrée en viticulture, Bordeaux, France : Éditions Féret (2003)

[2] C. David et coll., Agro-écologie, les mots de l'agronomie. $\mathrm{H}$

[3] FAO, Symposium international sur l'agro-écologie pour la sécurité alimentaire et la nutrition, Rome (2014) http://www.fao.org/about/meetings/ afns $/ \mathrm{fr} / ? \% \mathrm{EF} \% \mathrm{BF} \% \mathrm{BD} \% 04$

[4] M. Guillou et coll., Vers des agricultures doublement performantes pour concilier compétitivité et respect de l'environnement, INRA/AGREENIUM (2013) http: //agriculture.gouv.fr/remise-durapport-sur-lagro-ecologie-par-marionguillou-stephane-le-foll-0

[5] J. Loyat, Contribution de l'agriculture au développement durable. Recommandations pour la recherche, Natures Sciences Sociétés 15, 186-190 (2007)

[6] Ministère de l'agriculture de l'agro-alimentaire et de la forêt, Plaquette : Projets agro-écologique pour la France, (2013) http://agriculture. gouv.fr/ministere/projet-agro-ecologiquepour-la-france
[7] Ministère de l'agriculture de l'agro-alimentaire et de la forêt, Plaquette : Une première application de la loi d'avenir pour l'agriculture, (2016) http://agriculture.gouv.fr/sites/minagri/ files/giee-laaf-v4-bd.pdf

[8] J. Rochard, Un nouveau support de communication : l'éco-œenotourisme, paysage, biodiversité, écoconception des caves, 38th World Congress of Vine and Wine, OIV (2015)

[9] J. Rochard, B. Porte, Biodiversity and viticulture: concept and application; results of the european project biodivine, 37th World Congress of Vine and Wine, OIV (2014) http://agriculture. gouv.fr/sites/minagri/files/giee-laafv4-bd.pdf

[10] G. Sentenac et coll., Les bonnes pratiques pour favoriser les auxiliaires et préserver la biodiversité, numéro spécial ugvb (2015)

[11] TP. Tomich, S. Brodt, H. Ferris, R. Galt, WR. Horwath, E. Kebreab, J. Leveau, D. Liptzin, M. Lubell, P. Merel, R. Michelmore, T. Rosenstock, K. Scow, J. Six, N. Williams, J. Yang, Agroecology: A Review from a Global-Change Perspective. Review in advance, Sous presse (2011)

[12] A. Wezel, S. Bellon, T. Doré, D. Vallod, C. David, Agroecology as a science, movement or practice. Agronomy for Sustainable Development, 29 : 503-515 (2009)

[13] A. Wezel, JC. Jauneau, Agroecology - interpretations, approaches and their links to nature conservation, rural development and ecotourism. In: Campbell W.B., López Ortiz S., eds, Integrating agriculture, conservation and ecotourism: examples from the field. Issues in Agroecology - Present Status and Future Prospectus 1, Springer, Dordrecht (2011) 